\title{
GENETIC CONTROL OF THE TRIGGER FOR THE G2/M CHECKPOINT
}

\section{Award: DE-PS02-08ER08-20}

\section{Final report}

The current award is divided into two prinicpal components regarding effects stemming from acute low dose radiation. The first is the investigation of the threshold of activation of radiation induced cell cycle arrest and a mechanistic assessment of various radiosensitizing genes known to be involved in this important process. The relevance for this part stems from the prudent application of the Linear No-Threshold model as it informs widely employed radiation protection standards. The second part of the award is the validation of the participation of micro RNA (miRNA) molecules in radiation bystander effect biolgy in which an irradiated cell transmits damage to a non-irradiated bystander cell. This work involved generation of a new technology for isolation and manipulation of individual cells with absolute fidelity to allow for microbeam irradiation of a donor cell and the demonstration that a miRNA molecule is transferred to a particular recipient cell and imparts a biological effect on the recipient. The relevance here is further support of the bystander hypothesis with mechanistic insight as to how damage information is promulgated at the cellular level.

\section{Part 1: Mechanistic studies of the G2/M checkpoint and its components. (This work has been published in Radiation Research. 2012 June; 177(6): 743-750.)}

Maximum permissible doses, together with their rationale in terms of cancer risk, assume that the human population is homogeneous in radiosensitivity, with the exception of individuals such as 
ATM homozygotes who are too few in number to affect risk estimates and anyway are readily identified by their clinical symptoms.

Much of the radiation exposures accumulated by radiation workers correspond to doses below the level at which there is direct epidemiological evidence of an excess cancer incidence from, for example, the A-bomb survivors. Assessing risks at these doses is a contentious issue. The BEIR VII committee of the National Academy of Sciences, as well as ICRP, NCRP and UNSCEAR all recommend that for radiation protection purposes it is prudent and conservative to adopt the Linear, no-threshold model, which assumes that risk is proportional to dose and that there is no dose below which the risk is zero. Using this model, risks at low doses are estimated by a linear extrapolation from the higher doses where epidemiological data are available.

Evidence has accrued for a difference in radiation response biology for high doses as compared to low doses. As such, different radiation response factors could be involved in the activation in the prominent cell cycle arrest response. The human population is not genetically homogeneous and haploinsufficiency in one or two key radiation resistance genes could alter the lower end of the dose response curve.

\section{Experimental design}

The goals of this work were to assess rapidly dividing, minimally perturbed cell populations with combined haploinsufficiency for ATM, Mrad9 and PTEN, for their ability to halt at the $G_{2} / M$ checkpoint after irradiation with low and high doses. Various combinations of genetically haploinsufficient cells were generated by pairing mice of varying genotype and establishing mouse embryo fibroblast (MEF) cultures with desired genetic background. We tested whether the radiosensitivity seen to accompany combined haploinsufficiency is coincident with the checkpoint control functions of these genes. In the context of partially ablated checkpoint components in various combinations, we also sought to gain more detailed mechanistic insight into checkpoint function - especially at low doses. 


\section{Findings}

When normal cells are exposed to a dose of radiation large enough to cause chromosome aberrations, they become arrested at the G2/M checkpoint, and this delay allows repair of damage before the complex task of mitosis is attempted. It has been known for many years that cells homozygous for mutations in the ATM gene possess neither a competent G2/M checkpoint nor proficient repair of DNA damage. Similar trends are observed with other radioresistance genes forming the basis for their inclusion in our work:

ATM (ataxia telangiectasia mutated) is a protein kinase that, among its many roles, is involved in mediating DNA DSB repair. In addition to its DNA damage sentinel function, ATM is involved in multiple pathways regulating checkpoint control at the G1/S and G2/M transition. In the presence of DNA DSB, it is activated by autophosphorylation and it, in turn, phosphorylates a number of substrates involved in DNA repair, damage signaling, induction of apoptosis and cell cycle checkpoint control. (Shiloh and Ziv 2013)

Many of these substrates co-localize together and accumulate at sites of DSB, termed foci, which can be visualized by immuno-histochemical methods. These foci may represent sites of ongoing DNA repair or may also indicate checkpoint control points. In normal cells, with time, there are a decreased number of foci, representing completed DNA repair. In DNA repair deficient cells, high levels of remaining foci are frequently noted. ATM -deficient cells are hypersensitive to radiation due likely to a combination of defective checkpoint signaling and impaired DNA DSB repair.(Nelms, Maser et al. 1998)

RAD9 is an important component of cell cycle checkpoint control, DNA damage recognition, regulation of apoptosis and maintenance of genomic integrity. In response to ionizing radiation, ATM induces RAD9 hyperphosphorylation. Homozygous Mrad9 knockout cells are highly sensitive to ionizing radiation and also show defects in the maintenance of G2/M checkpoints. (Lieberman 2006)

PTEN, a tryrosine phosphatase with activity against PI3-kinase phosholipid and some protein substrates is best known for its role as a tumor suppressor, much like p53, but also appears to be involved in maintenance of chromosomal integrity. Homozygous deletions in PTEN are embryonic lethal but almost $90 \%$ of mice heterozygous for PTEN survive for at least 56 weeks. 
PTEN $^{-1-}$ cells exhibit a partially defective checkpoint response and genetic instability after exposure to ionizing radiation PTEN is upregulated by EFR1 after gamma irradiation but also appears to be transcriptionally regulated by p53 PTEN and p53 appear to regulate each others activities in several different ways. (Bassi, Ho et al. 2013)

The impact of low and high doses of radiation on cell cycle progression was studied in actively cycling MEFs. Doses of 10, 50 and 100 mGy were used for the low dose experiments, and doses of $0.5,1.0$ and $5.0 \mathrm{~Gy}$ were used for high dose studies. Fibroblasts were seeded, permitted to recover and then subjected to irradiation and fixed for cell cycle analysis. Wild type MEFs isolated from heterozygous Atm pairings recapitulated the 6-8 hour maximal arrest with 5.0 Gy while lower 0.5 and 1.0 Gy doses caused a slight shift in the curve to a maximal arrest closer to 6 hours. Cells derived from Atm null embryos failed to mount a robust $\mathrm{G}_{2} / \mathrm{M}$ arrest in response to 5.0 Gy irradiation. Interestingly, and, counter to observations in other biological systems we have studied, no intermediate phenotype was seen in heterozygous MEFs haploinsufficient for ATM for the G2/M arrest endpiont.

Given that lower doses generated maximum arrest responses at 6 hours post irradiation, detailed low dose comparisons over the range of 0-100 mGy were conducted at this time point. In accord with trends observed at higher doses, checkpoint induction was absent in MEFs null for Atm over this low dose range. Importantly, no significant increase in $\mathrm{G}_{2} / \mathrm{M}$ fraction was observed in wild type MEFs or those haploinsufficient for ATM. Cells with combined haploinsufficiency for ATM and PTEN showed no significant induction in the $G_{2} / M$ population over the low dose range of interest. Throughout the time courses, $\mathrm{G}_{1}$ and $\mathrm{S}$ phase populations were also examined, but the most robust findings were seen at the $\mathrm{G}_{2} / \mathrm{M}$ checkpoint.

To further probe differences in checkpoint function, doses between 0.5 and 5.0 Gy were used on MEFs with combined haploinsufficiency for PTEN, ATM and Mrad9 based on previously demonstrated known interactions with the $\mathrm{G}_{2} / \mathrm{M}$ checkpoint apparatus. Wild type MEFs derived from littermates over various $F_{1}$ pairings reproducibly mounted a characteristic 6 hour maximum arrest, except when doses increased to 5.0 Gy where the arrest maximum was delayed until 8 hours post irradiation and resolution of the arrest did not complete by the end of the 12 hour time course. MEFs that were combinatorially haploinsufficient for two radioresistance proteins exhibited no significant deviation as compared to MEFs from wild type littermates. 
We observe statistically significant increases in the $G_{2} / M$ fraction of MEFs doubly haploinsufficient for ATM and PTEN at doses above $0.5 \mathrm{~Gy}$. We do not see a statistically significant change in the $\mathrm{G}_{2} / \mathrm{M}$ fraction over the dose range of 0-100 $\mathrm{mGy}$. This threshold for activation between 100 and $500 \mathrm{mGy}$ is also observed by other laboratories studying checkpoint function (Xu, Kim et al. 2002; Jeggo 2010). This same threshold also seems to represent the lowest threshold of damage that is tolerated for subsequent release from the checkpoint (Deckbar, Birraux et al. 2007; Lobrich and Jeggo 2007). The significant and unique finding presented here is that the activation of all cell cycle checkpoints occurs irrespective of haploinsufficiency contributed by various combinations of radioresistance genes when examined in freshly isolated MEFs after acute exposures to sufficiently high doses gamma radiation. The $\mathrm{G}_{2} / \mathrm{M}$ checkpoint, irrespective of haploinsufficiency in MEFs, engages at a threshold greater than $0.1 \mathrm{~Gy}$ and less than $0.5 \mathrm{~Gy}$.

Our findings suggest that there is not a discernible connection between the radiosensitivity that arises from haploinsufficiency in our other systems of study and the control of the $G_{2} / M$ checkpoint. Moreover, a substantial dose, coincident with a significant amount of DNA damage, must be applied for activation of the checkpoint. This means that there is essentially no detectable checkpoint at low doses. Activation of the checkpoint only occurs at doses where most of the cells, bound for mitotic catastrophe, are reproductively dead. Lastly, our findings confirm a threshold of activation for the $\mathrm{G}_{2} / \mathrm{M}$ checkpoint, hinting at two separate radiation response programs acting below and above this threshold.

\section{Part 2: Investigation of miRNA transfer as an underlying factor in Radiation bystander} effect biology. (This work is in press in Journal of Micromechanics and Microengineering, 23 (2013) 000000 (8pp).

A central element of this work has been the development of a cell sorting techology to correctly partition irradiated and non-irradiated cells with absolute fidelity. Several methods are currently available to sort single cells. These methods offer varying sorting efficiency, rate, and batch size contingent on the physics of the separation method. Historically, sorting of cells from small cell populations had been undertaken using several approaches. Single cells can be sorted manually 
by a skilled operator using micropipettes, microgrippers or size-based filters (Yi, Li et al. 2006). The typical sorting frequency with these methods is very low $(\sim 1 \mathrm{cell} / \mathrm{min})$ and the physical manipulation may cause damage to the cell. Also, single cell manipulation can be achieved by integrating microfluidics with magnetic, optical, electrical, or hydrodynamic forces.

We designed, constructed and optimized the use of a manually operated microfluidic cell sorter that 1) can accurately sort single or small groups of cells from very small cell populations with minimal losses, 2) that is easy to operate and that can be used in any laboratory that has a basic fluorescent microscope and syringe pump, 3) that can be assembled within minutes, 4) that can sort cells in very short time (minutes) with minimum cell stress, 5) that is cheap and reusable. The operation and use of the sorter can be reviewed in an online training video available at the link here: (http://bit.ly/1fMG0ZH).

Experiments have been completed showing that cells are very gently handled with the technology with a measured mortality increase of $3 \%$ as compared to controls. Further, a novice operator was able to successfully sort live cells in 9 of 10 iterative runs conducted in a span of less than $3 \mathrm{~h}$.

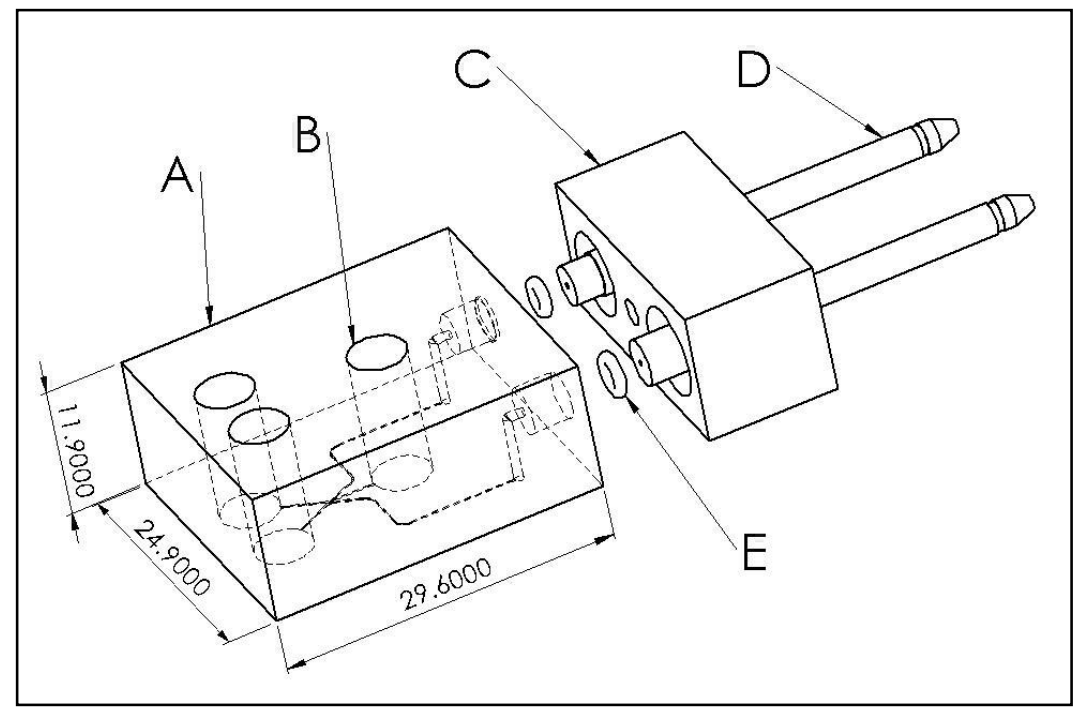

Figure 1. Principal components of the micofluidic cell sorter. The main body (A), inlet reservoir (B), valve-containing body (C), valves (D) and seals (E). 
Separation and isolation of irradiated and non-irradiated cohorts is a critical capability for correct demonstration of changes in donor and recipient cell biology and so the establishment and validation of this sorting technology is a great addition to the laboratory for this and other studies of bystander effect biology. The addition of this sorting capability will be brought to bear in future microbeam irradiation studies of investigating bystander effect biology.

\section{Part 3: Radiation induced cell to cell miRNA transfer.}

\section{Introduction}

MicroRNAs (miRNA) are 21-23 mer RNA molecules which are essential for organism development and cell functions. They regulate gene expression by binding to the 3'UTR inducing either miRNA degradation or mRNA silencing. The most characteristic properties of miRNA are their multi-targeting potential (one miRNA may target many genes and one gene may be targeted by many miRNA) and the modest down regulation of their targets (in the range of 1.5, 2 fold). Therefore miRNA acting in parallel promote massive fine tuning of gene expression (Bartel 2009). Currently, it is well established that different cell types and tissues have specific miRNA expression profiles, that miRNA signatures are disease-specific and may report the type and stage of some diseases (Farazi, Hoell et al. 2013). The importance of miRNA expression profiles in disease raised the question as to what extent they may play role in cell to cell communications. In plants, for example, intercellular miRNA appear to act as long distance signaling molecules by down regulating specific targets (Mallory and Vaucheret 2006). Although miRNA systemic response in animals is still not confirmed, miRNA are secreted into the extracellular space as part of the content of the exosomes and microvesicles (Kosaka, Yoshioka et al. 2013). Since it was shown that proteins contained in the microvesicles are actively involved in modulation of the activities of the cells that endocytose them, an important question is to what degree miRNA are involved in these processes. Such transfers by vesicles have been shown to be a component of immune function, specifically there is a unidirectional transfer of miRNAs from the $\mathrm{T}$ cell to antigen presenting cells, mediated by the delivery of 
CD63+ exosomes at the immune synapse (Mittelbrunn, Gutierrez-Vazquez et al. 2011). The later finding highlights the possibility for transfer of functional miRNA between human cells.

We hypothesize that functional miRNA transfer between cells may occur in radiation induced stress conditions as part of a stress response in cell signaling. Since radiation is widely used in tumor treatments, the study of its effects may shed light on the mechanisms of cell response to radiation. Based on the significant regulatory capacity of miRNA, the importance of such mechanism could be great.

To test our hypothesis we focused our studies on showing is there miRNA transfer between normal human cells and that this transfer is stress dependent.

We used two experimental systems. In the first one we studied miRNA transfer between cocultures of 1) cells expressing miRNA specific for the nuclear membrane protein lamin and 2) normal cells. The readout was the decrease of lamin expression in cells adjacent to the lamin miRNA expressing cells determined by quantitative confocal microscopy. In the second system, we studied miRNA transfer between cocultures of 1) cells where the expression of GFP and RFP is regulated by switch mechanism consisting of the bacterial $\mathrm{CuO}$ operon and 2) cells expressing shRNA that may turn this switch to on state leading to the expression of GFP and RFP. Here the readout is GFP/RFP expression determined by flow cytometry.

\section{The description of the results from both systems follows.}

\section{System 1: Cell to cell transfer of functional miRNA specific for the nuclear protein lamin.}

The principle of this assay is relatively simple. Normal human fibroblasts (AG01522 cells) engineered to express GFP and shRNA against the nuclear protein lamin were mixed and cultured together with normal human AG01522 fibroblasts. Lamin expression in all cells is measured after irradiation followed by comparison of lamin expression between cells neighbor and non-neighbor to the GFP/shRNA lamin expressing cells. The lamin miRNA expressing cells can be clearly identified based on the GFP expression. The idea is that an exchange of miRNA against lamin between the lamin miRNA expressing cells and their neighbors will reduce the lamin expression in the later. Lamin expression will not be reduced in cells which are not in contact with the miRNA expressing cells. 
For the assay, a mixed culture of both cells types is maintained for 2-4 days. Some of the cultures are treated with radiation and some are used as non-irradiated controls. The cells are fixed at preselected time points and then are stained for the nuclear protein lamin using an anti-lamin antibody. The relative brightness of the staining intensity of of adjacent and the non-adjacent cells of the lamin specific miRNA is estimated by quantitative confocal microscopy. We encountered 3 main problems during the development of this system. The first was to obtain stably transfected cell clones. Since for the experiments we used normal human cells, (the normal human fibroblasts AG01522), the transfection (with non-viral vectors) and the isolation of single clones was formidable task due to the very small frequency of efficient vector DNA integration in to the cell genome. We overcome this problem by using very early passage of AG01522 cells (passage18) and by selecting (with blasticidin) large numbers of cells. The second problem was related to the high complementarity between the lamin miRNA and the lamin mRNA. Lamin was significantly reduced in the lamin miRNA expressing cells which in most cases led to destabilization and apoptosis of transfected cells. As a result there were very small number of clones where the expression of lamin was not dramatically reduced and the cell functions were normal. These cells were used for the coculture experiments. A third problem was related to the quantification of the lamin expression. For this, lamin in cells was stained with anti-lamin Alexa-Fluor 594 labeled antibody and scanned by confocal microscope for detailed estimation of the distribution of the stained lamin. To properly quantify this staining and to allow comparison between different cells in the same field and between different samples the brightness data needs to be normalized to some universal reference standard. We found that the field's background brightness could not be used since it was uneven when we carefully tested our confocal microscopes (a Nikon and Zeiss systems). To overcome this problem, beads with uniform brightness were embedded each sample during slide mounting. The brightness of these beads was used to normalize the brightness of the staining in the single samples and between samples as elaborated in the methods below. 


\section{Materials and Methods for System 1}

\section{GFP/Lamin expressing cells}

Cells: Normal human fibroblasts AG01522 cells were purchased from Coriell Institute for Medical Research at passage 16 . The cells were expanded to passage $18^{\text {th }}$ and after that used for transfection.

\section{Lamin specific miRNA expressing vector: pcDNA $^{\mathrm{TM}} 6.2-\mathrm{GW} / \mathrm{EmGFP}-\mathrm{miR}-\mathrm{LMNA}$} miRNA Vector (Invitrogen) was used for cell transfection. The vector contains a $68 \mathrm{bp}$ insert which encodes a small hairpin precursor of miRNA specific for the Lamin an mRNA. It also has coding sequence for EGFP driven by the same promoter. The expression of EGFP allows the detection of the cells expressing the lamin specific miRNA by fluorescent microscopy. A map of the vector is shown on Figure 2.

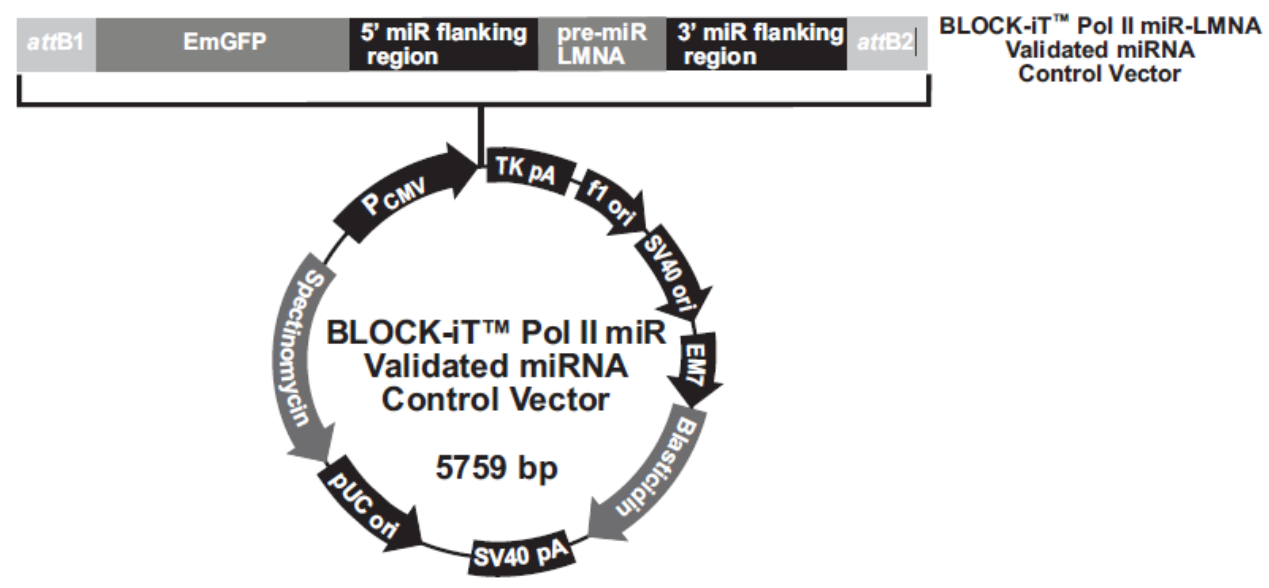

\section{Cell transfection and clone isolation:}

AG01552 cells were plated at density of $3 \times 10^{4}$ cells $/ \mathrm{cm}^{2}$ in $10 \mathrm{~cm}$ plates and incubated for 24hrs. The cells were transfected with 20ug of pcDNA ${ }^{\mathrm{TM}} 6.2-\mathrm{GW} / \mathrm{EmGFP}-\mathrm{miR}-\mathrm{LMNA}$ miRNA Vector using Lipofectamine 2000. Two approaches for the isolation of GFP/Lamin miRNA positive clones were used. The first one involved sorting of the transfected cells 2 days after transfection, culturing for 3 more days followed by plating of the cells at density of $50 / \mathrm{cm}^{2}$ and selection of the GFP expressing cells by blasticidin( $8 \mathrm{ug} / \mathrm{ml}$ )(Invitrogen) for 10 days. In the second approach the transfected cells were trypsinized four days after the transfection and plated at density of $350 / \mathrm{cm}^{2}$ in $10 \mathrm{~cm}$ plates in media containing blasticidin (8ug/ml). In both 
approaches GFP positive clones were isolated ten days later using cloning cylinders and expanded in 12 well plates. A small numbers of these cells were plated in 4 well chamber slides and stained for Lamin for evaluation of the Lamin A expression. The clones show different degree of lamin A expression and the one where the lamin A expression was reduced the most were used for experiments.

\section{Cell cocultures}

A mix of cells containing $4.10^{5}$ AG01522 cells and 2.10 3 GFP/miR-Lamin A expression cells (ratio 1:200) were plated in a wells of 2 well chamber slides and cultured for 24 hours. After that, half of the slides (typically 4) were irradiated with 5Gy of $\square$-rays, cultured for 36 hours and fixed and stained for Lamin A. Before mounting, the slides were overlaid with mounting media containing Dynabeads Protein A (Life Technologies, Grand Island, NY 14072)) which were used as fluorescence calibrator for the confocal microscopy.

Lamin Staining: The cells were fixed in 4\% paraformaldehyde and stained with anti-lamin rabbit antibody labeled with Alexa Fluor 594. The slides were mounted using aqua mount medium.

\section{Quantitative confocal microscopy}

The Lamin A stained samples were observed on Nikon T2000 confocal microscope under the control of EZ-C1 software. Separate images of the green channel (for GFP) and of the red channel (for Lamin staining) were collected. Total of 10 image planes were taken from each filed of observation which included the whole cell volume. These planes were then assembled in one image using the "volume render" option of the software. These images were exported to Photoshop. The position of the GFP/Lamin specific miRNA expressing cells and their neighbor cells was determined by overlaying both green and red images. After numbering the average brightness of each nucleus was determined in Photoshop by using the histogram tool. These values were recorded in Excel and were subject to statistical analysis. 


\section{Calibration of the microscope field and lamin expression comparison:}

To avoid false estimation of lamin staining, Protein A beads (Dynabeads, Life Technologies, Grand Island, NY 14072), were used. The beads emit very uniform fluorescence at 600-650 nm which is the range where we recorded the lamin staining. The beads were prepared by three washed with PBS and added to the mounting media. For the estimation of the uniformity of the image, the microscopy field was divided into 9 quadrants. The brightness of the beads in the different quadrants was estimated using the histogram tool of Photoshop and these values were statistically compared between the different quadrants by t-test. Only quadrants where there was no statistically significant difference of the beads brightness were used for lamin expression determination and for data collection.

\section{Results:}

\section{Cell transfection:}

Total of 15 GFP/ Lamin A specific miRNA expressing clones were isolated in three transfection experiments following the methodology described above. Once isolated by cloning cylinders, they were expanded in 12 well plates. These clones had different degree of lamin expression in comparison with the wild type cells, varying from very low (20\%) to high (80\%). For the cell mixing experiments we used only cells with very low expression of lamin A, assuming that these cells have high expression of lamin a specific miRNA.

\section{Calibration of the microscope field.}

Dynabeads were used for selection of comparable fields in the same and different experiments. The beads have very uniform fluorescent expression in the range of the anti-lamin antibody fluorescence which was labeled with Alexa Fluor 594nm. For the imaging measurements, the brightness of beads from different pictures was compared. Only images where the bead brightness show no statistically significant difference were used for lamin expression data determination (fig.1) 


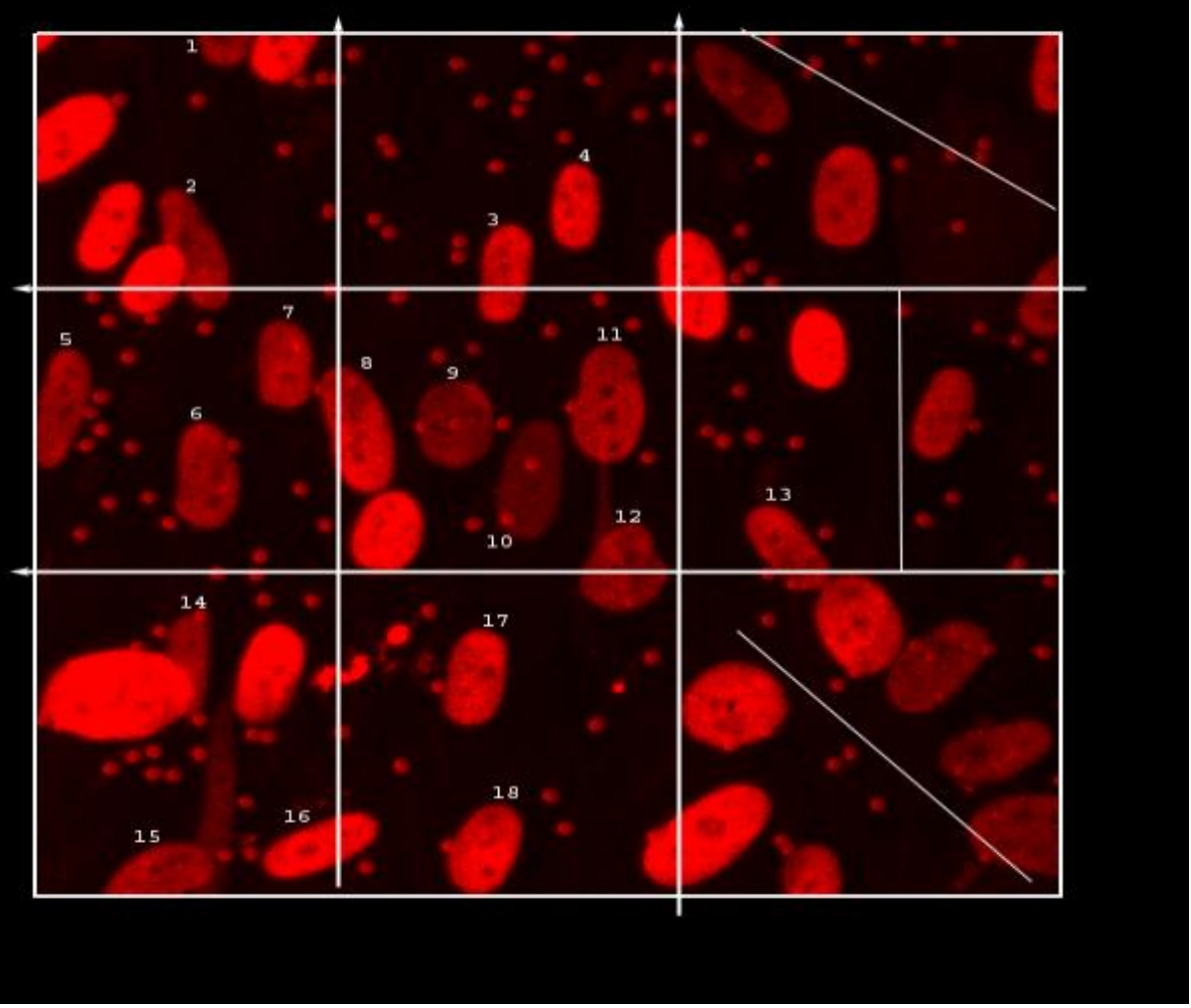

Figure 3. Dynabeads embedded between cell nuclei stained for lamin A. The brightness of the beads from each quadrant were compared and used as reference for validation of the lamin brightness. Lamin brightness in each of the numbered nuclei stained for lamin was used for lamin brightness estimation.

\section{Cocultures and lamin expression comparison.}

AG01522 cells expressing lamin specific miRNA were cocultured with normal AG01522 cells. Images like the one shown in Fig.2 were used to determine the cells that are in contact with the lamin miRNA expressing cells (designated as neighbors) and cells that are not in contact with the miRNA expressing cells, designated as non-neighbors. 

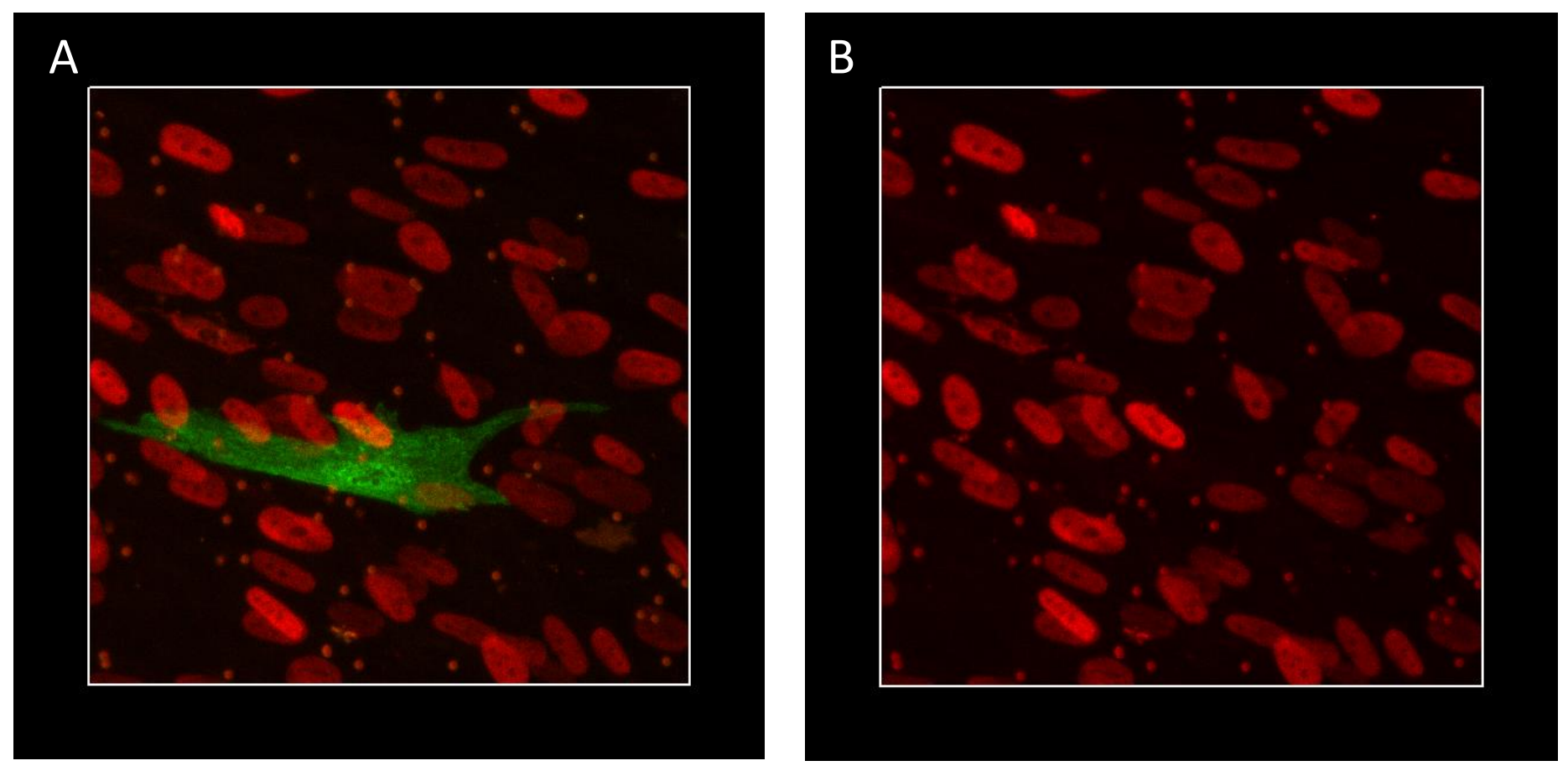

Figure 4. For estimation of the neighbors of the miRNA expressing cell, an images of the both green and red channel as shown in A were used. Cells that were in contact with the green cells in A were designated as neighbors. All other were considered non-neighbor cells. The brightness of each nucleus was measured in the single channel images shown on B.

Data for cell brightness of neighbor and non-neighbor cells was obtained from of four miRNA transfer experiments. A total of 486 neighbor cells to the Lamin specific miRNA expressing cells and 661 non neighbor cells were measured. From the neighbor cells, 235 were irradiated neighbors and 251 were non-irradiated neighbors. The data for the brightness of all cells was compared for each experiment separately on neighbor vs. non-neighbor basis by ANOVA and $t$ test. In three of these experiments there was detectable difference between the brightness of neighbor vs. non-neighbor cells. In one experiment we didn't detect such differences. A summary of the positive experimental results is shown on fig.3. 
A) Non-irradiated cells

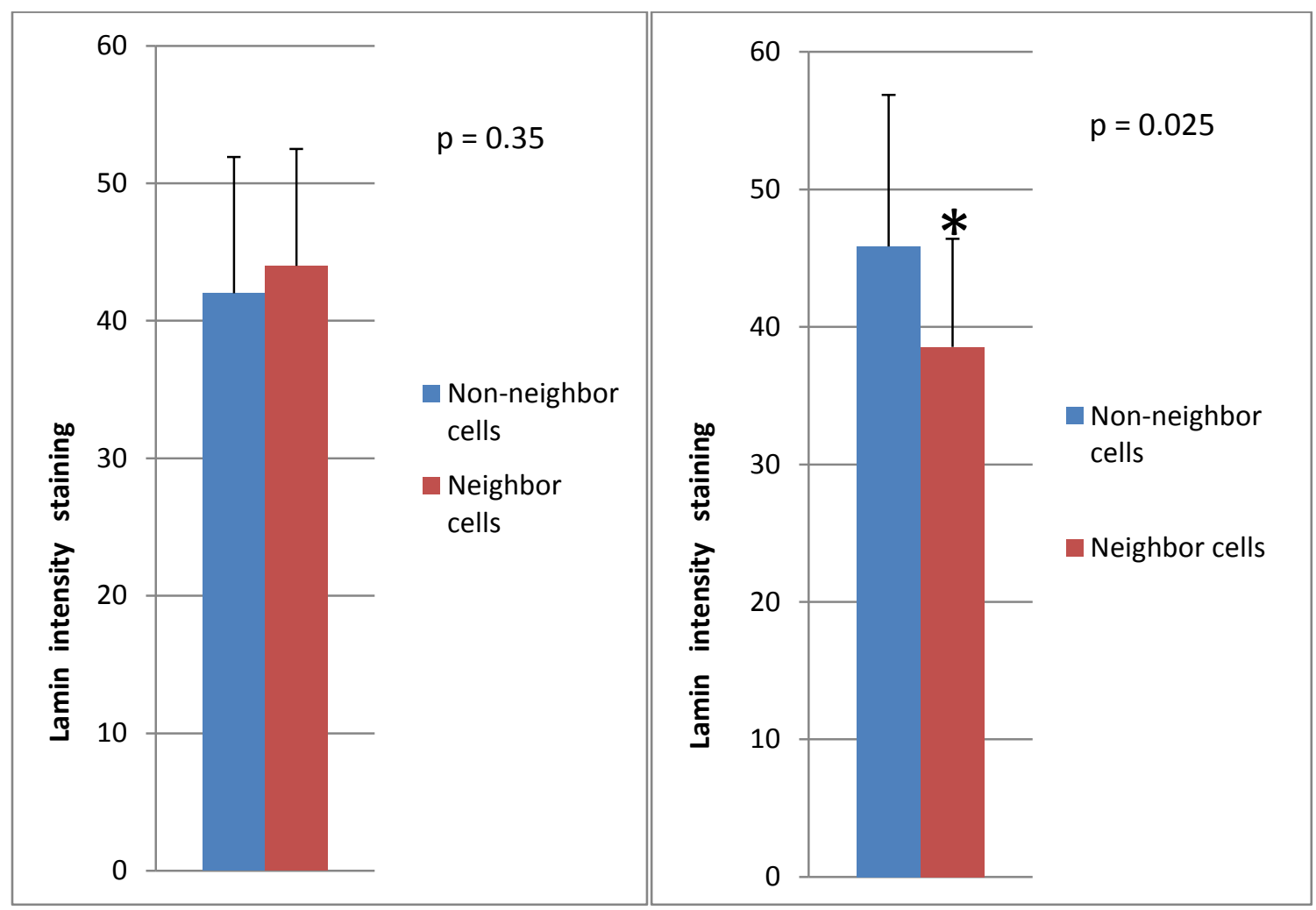

Figure 5. Difference in lamin intensity in neighbor vs. non neighbor cells to the lamin miRNA expressing cells. A statistically significant reduction of the lamin A expression levels was detected in the irradiated neighbors of the lamin A specific miRNA expressing cells indicating that upon radiation exposure, functional miRNA was transported between the GFP/miR-lamin A expressing cells and neighboring non expressing cells. No significant difference of lamin A expression was detected in the non-irradiated cells.

These results show statistically significant differences in the brightness between irradiated neighbor and non-neighbor cell which indicates that cells under stress (in our case induced by high dose of radiation) may exchange functionally active miRNA by either direct or indirect mechanism. Such transfer was not found in the non-irradiated cells. 


\section{Conclusion:}

We generated stable cell clones of normal human fibroblast expressing GFP and lamin a specific miRNA. This miRNA reduced significantly the expression of the endogenous lamin A. Cocultures of these cells with non-transfected cells show that lamin expression was reduced in irradiated neighbors of the GFP/miR-lamin expressing cells suggesting that functional lamin specific miRNA was transferred between the miRNA producing and non-producing cells. This effect was not detected in non-irradiated cells. These results suggest that stress may be an important factor in cell to cell transfer of miRNA.

\section{Limitation of the system:}

A notable limitation of the described system was that the detected differences in the lamin expression of the neighbor cells was relatively low and could be established only after statistical comparison but not by direct observation. This led us to rethink this approach and to develop a different system where the fluorescence could be induced (rather than reduced) and where it could be measured directly in live cells without using antibodies. Fluorescent proteins were an obvious choice for such system and we decide to use a switchable scheme where the expression of GFP and RFP may be induced by miRNA.

\section{System 2: Engineering a molecular trigger driven by shRNA molecule.}

As outlined above, the key element to the miRNA silencing assay involved elimination of lamin expression in the putative recipient cell population. We sought to reverse this by devising a system in which the small RNA transfer event is reported by activation or induction of an event in the system that was not present before. In this "de-repression" strategy we used elements of an unrelated plant cell signaling system in which transcription becomes activated after a small molecule is added to the system. In this system, the small molecule cumate binds to and inactivates the CymR promoter repressor. The CymR repressor is then released from its binding site in the cumate operator $(\mathrm{CuO})$ allowing for transcription of the downstream GFP and RFP genes. (Figure 6). There are important advantages to this strategy which include the 
"tightness" of the control of expression from this molecular switch, its commercial availability in a reliable viral vector format and the fact that the plant signaling components involved in small molecule sensing would be unaffected by radiation. In this way, a triggering system could be easily assembled.

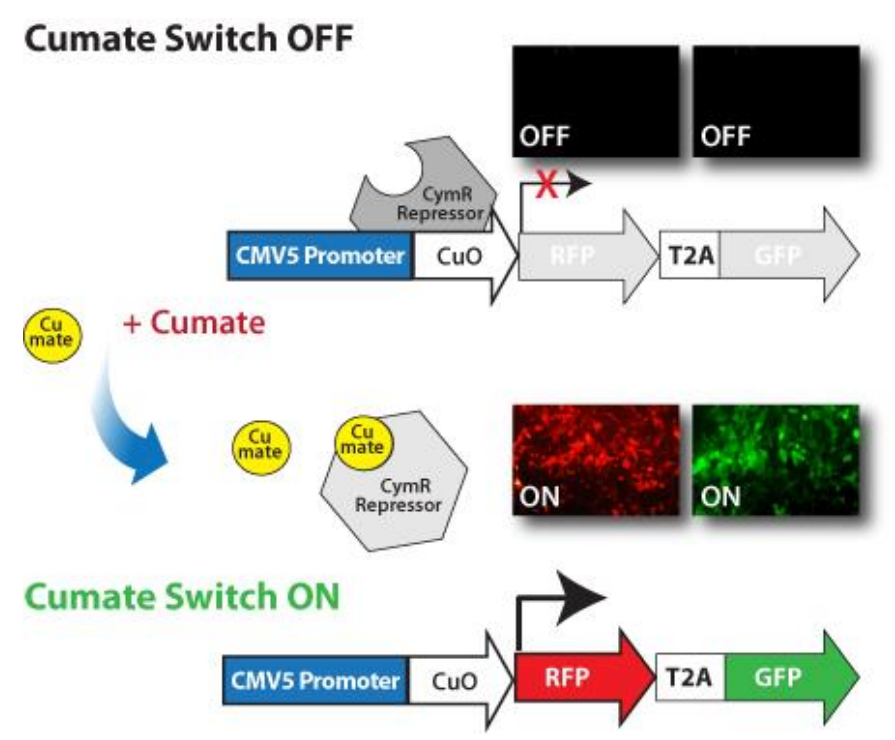

Figure 6. Elements of the commercially available cumate switching system.

Fibroblast cell lines are commonly employed in radiation biology and specifically in radiation induced bystander effects since they are relatively robust and divide very actively (http://bit.ly/1fuH2M7). Bystander responses have been observed also in most cell types, including lymphocytes, endothelial cells and tumor cells (Prise and O'Sullivan 2009). For the following experiments we chose to use endothelial cells specifically because they show prolonged and elaborate cell to cell contacts in culture (http://bit.ly/15yMX9a) which could enhance small RNA transfer. 


\section{Methods}

Cell Culture - CRL 4025 TIME cells were obtained from the ATCC (Manassas, VA 20110 USA) Cells were maintained in humidified incubator at $37^{\circ} \mathrm{C}$ and $5 \% \mathrm{CO}_{2}$ and were fed three times per week with EGM-2 media supplemented with Penicillin/Streptomycin and Geneticin.

Generation of a Reporting system for miRNA transfer - Recipient Reporter cells were generated by infecting CRL4025 cells (TIME cells XX) with lentiviral particles encoding expression of both RFP and GFP under the regulation of a cumate binding operator sequence (SBI, Mountain View, CA 94043). A stably expressing clone was then superinfected with a second lentivirus providing expression of the CymR repressor. Recipient Reporter cells were not fluorescent but were induceable with cumate at $10 \mathrm{ug} / \mathrm{mL}$. The Donor cells, were engineered to express shRNA targeting the CymR repressor. The sequence for the cumate repressor was submitted to Sigma-Aldrich (St. Louis, MO 63178) algorithm service for generation of three candidate siRNA molecules which were transiently transfected with cationic lipid reagents. One candidate, with the sequence 5'- CGACCGGGAUCCAGCGUUAUU-3' was found to induce GFP expression in the Reporter cell line. This sequence was packaged into MISSION shRNA expression lentiviral particles which were used to infect naive parental CRL4025 TIME cells generating cells stably expressed the siRNA. Insertion was verified by PCR and Sanger Sequencing.

The resultant small RNA transfer reporting system is shown in figure 5. It allows for assessment of the transfer of consitutively produced siRNA in the Donor cell line. There is also an allowance to check on the performance of the system at any time by addition of the cellpermeable small molecule cumate which will derepress the CymR protein generating a positive control signal in the system. The system further has the advantage of fluorescence reporting which can be assessed in populations via flow cytometry. Thus, if the signal is modest a shift in mean fluorescence intensity in the study population can be identified. 


\section{No transfer}

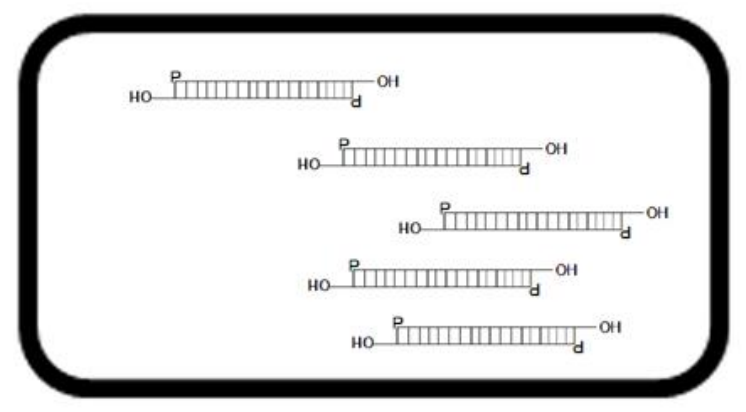

Donor

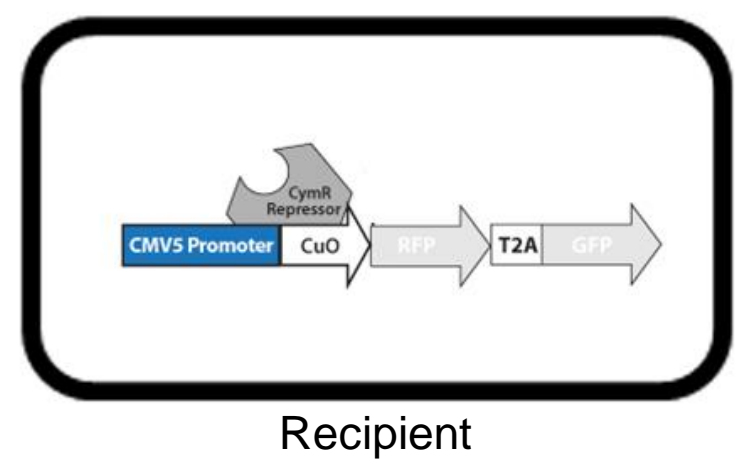

\section{Productive transfer}
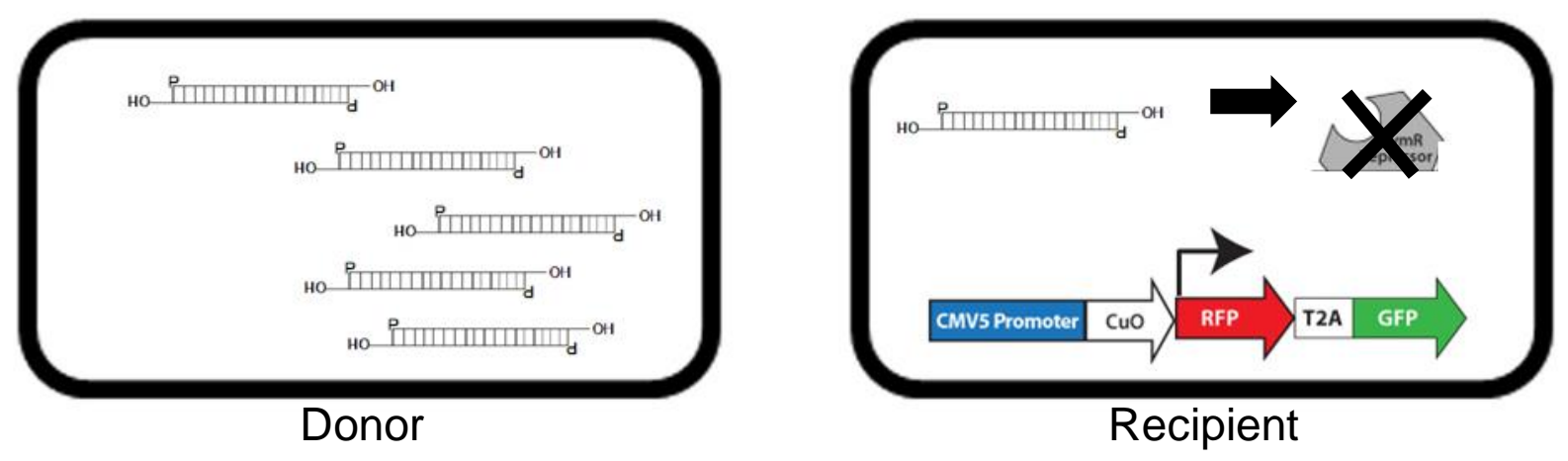

Figure 7. Small RNA transfer reporting system for use in endothelial cells. Transfer of siRNA targeting the CymR repressor decreases the expression of $\mathrm{CymR}$ which fails to bind to the $\mathrm{CuO}$ operator. The result is increased expression of GFP and RFP.

miRNA transfer assay - miRNA Recipient/Reporter cells and miRNA Donor cells were seeded in 24 well dishes at $39 \mathrm{kcells} / \mathrm{cm}^{2}$ at ratio 1:15 late in the day and allowed to spread and recover overnight. The following day, cells were irradiated with 5.0 Gy of g-rays in a Gammacell 40 apparatus (Atomic Energy, Ontario, Canada) that delivered ${ }^{137} \mathrm{Cs}$ rays at a 
dose rate of $0.79 \mathrm{~Gy} / \mathrm{min}$. Cells were fed with fresh media and returned to standard culture conditions. After this incubation, cells were trypsinized and subject to FACS analysis for determination of GFP expression relative to cumate treated and sham irradiated controls which were cultured in parallel.

\section{$\underline{\text { Results }}$}

Coculture experiments were conducted in which Donor and Recipient cells were allowed to interact for several time points after irradiation. Some of the results that we obtained using this system show and induction of GFP/RFP fluorescence in the Recepient cells which is an indicator for radiation induced transfer between cells (Fig.6).

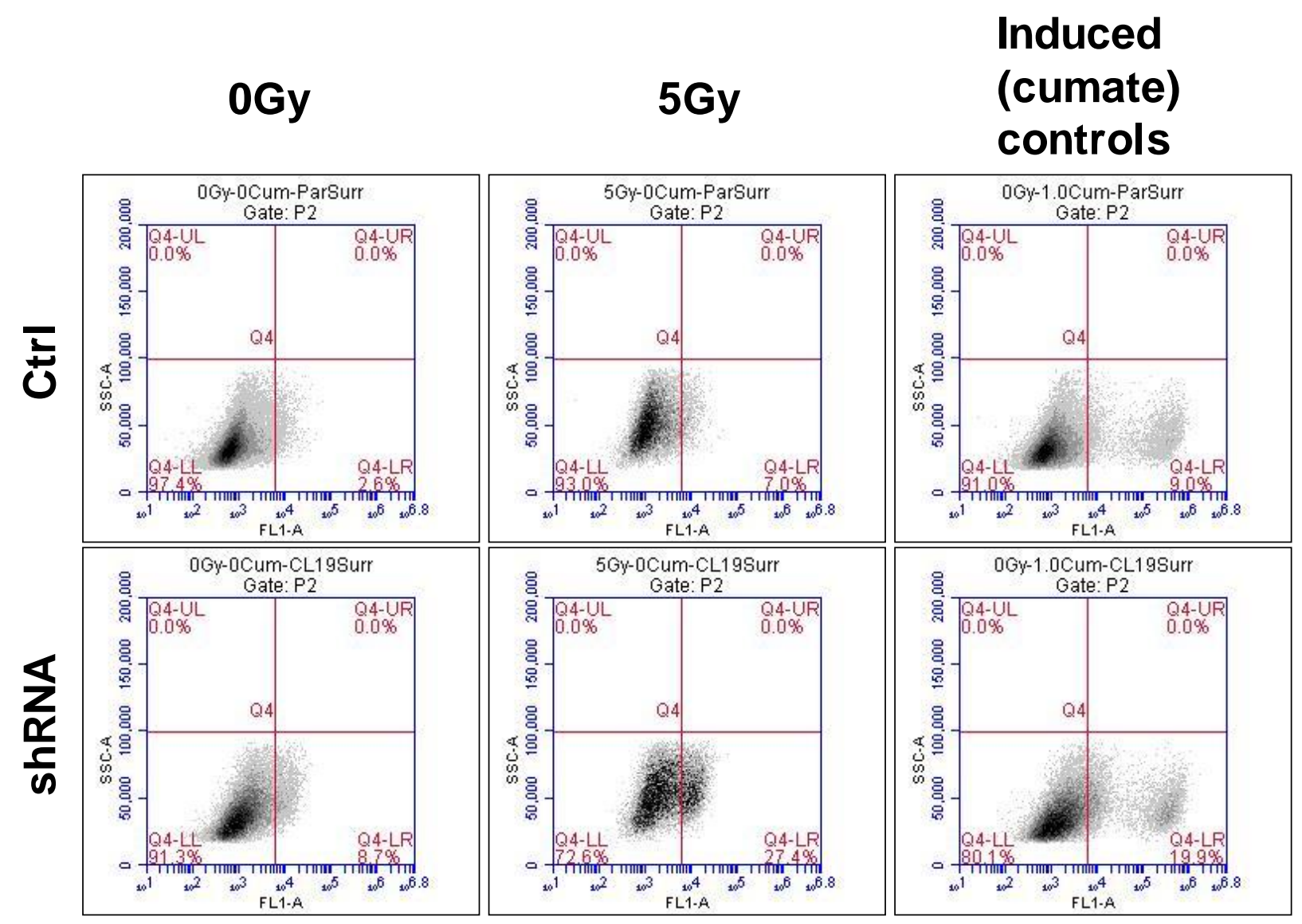

Figure 8. Radiation enhanced shRNA transfer. GFP reporting Recipient cells were cocultured with shRNA expressing Donor cells in a 1:15 ratio and incubated for $48 \mathrm{~h}$ after irradiation. Cells were then trypsinized and subject to flow cytometry analysis. Irradiated Recipient reporter cells 
exhibit increased fluorescence when surrounded by shRNA expressing Donor cells. This is not seen in non-irrdiated conditions and is also not seen with control Donor cells that do not express shRNA. The capability of the Recipient reporter cells to respond is demonstrated by greatly enhanced fluorescence of the Recipient reporter pool when cells are treated for 48h with $30 \mathrm{ug} / \mathrm{mL}$ cumate (right most panels).

\section{Conclusions}

Our small RNA transfer detection system is capable of reporting increases in GFP expression that seems to be enhanced with radiation as shown in Figure 6. The signal we obtain under these conditions is not robust and is not reproducible in half of our experiments.

The weak nature of the signal in several experiments is difficult to parse in the context of a radiation dependent change in our cells. In our studies to date, we have observed a change in cell autofluorescence in the FL1 and FL2 channels of our flow cytometer that is commensurate with radiation. This could be attributable to changes in the cytoskeleton of endothelial cells that are known to take place after irradiation (Young and Smilenov 2011). This change in autofluorescence has obscured subtle radiation induced differences in FL1 (GFP) intensity. Future plans involve quantitation of GFP by employing permeabilized flow cytometery with antibodies directed against GFP which are conjugated to fluorophores such as APC which emit in the FL4 channel of our instrumentation. Curiously, the FL4 detectors do not report radiation dependent changes in fluorescent intensity of our cells. These studies will be continued in the CRR/RARAF P41 study.

\section{References:}

Bartel, D. P. (2009). "MicroRNAs: target recognition and regulatory functions." Cell 136(2): 215-233.

Bassi, C., J. Ho, et al. (2013). "Nuclear PTEN controls DNA repair and sensitivity to genotoxic stress." Science 341(6144): 395-399.

Deckbar, D., J. Birraux, et al. (2007). "Chromosome breakage after G2 checkpoint release." J Cell Biol 176(6): 749-755.

Farazi, T. A., J. I. Hoell, et al. (2013). "MicroRNAs in human cancer." Adv Exp Med Biol 774: 1-20. 
Jeggo, P. (2010). "The role of the DNA damage response mechanisms after low-dose radiation exposure and a consideration of potentially sensitive individuals." Radiat Res 174(6): 825-832.

Kosaka, N., Y. Yoshioka, et al. (2013). "Trash or Treasure: extracellular microRNAs and cell-tocell communication." Front Genet 4: 173.

Lieberman, H. B. (2006). "Rad9, an evolutionarily conserved gene with multiple functions for preserving genomic integrity." J Cell Biochem 97(4): 690-697.

Lobrich, M. and P. A. Jeggo (2007). "The impact of a negligent G2/M checkpoint on genomic instability and cancer induction." Nat Rev Cancer 7(11): 861-869.

Mallory, A. C. and H. Vaucheret (2006). "Functions of microRNAs and related small RNAs in plants." Nat Genet 38 Suppl: S31-36.

Mittelbrunn, M., C. Gutierrez-Vazquez, et al. (2011). "Unidirectional transfer of microRNAloaded exosomes from T cells to antigen-presenting cells." Nat Commun 2: 282.

Nelms, B. E., R. S. Maser, et al. (1998). "In situ visualization of DNA double-strand break repair in human fibroblasts." Science 280(5363): 590-592.

Prise, K. M. and J. M. O'Sullivan (2009). "Radiation-induced bystander signalling in cancer therapy." Nat Rev Cancer 9(5): 351-360.

Shiloh, Y. and Y. Ziv (2013). "The ATM protein kinase: regulating the cellular response to genotoxic stress, and more." Nat Rev Mol Cell Biol 14(4): 197-210.

Xu, B., S. T. Kim, et al. (2002). "Two molecularly distinct G(2)/M checkpoints are induced by ionizing irradiation." Mol Cell Biol 22(4): 1049-1059.

Yi, C., C. W. Li, et al. (2006). "Microfluidics technology for manipulation and analysis of biological cells." Analytica Chimica Acta 560(1-2): 1-23. 